\title{
Common Sources of Bias in Gene-Lifestyle Interaction Studies of Cardiometabolic Disease
}

\author{
Tuomas O. Kilpeläinen
}

Published online: 29 September 2013

(C) Springer Science+Business Media New York 2013

\begin{abstract}
The role of gene $\mathrm{x}$ lifestyle interactions in the development of cardiometabolic diseases is often highlighted, but very few robustly replicated examples of interactions exist in the literature. The slow pace of discoveries may largely be due to interaction effects being generally small in magnitude and/or more complex than initially thought. However, the progress may also be hindered by the poor accuracy in large-scale epidemiological studies to estimate the true interaction effect sizes. Often, this bias tends to underestimate the interaction effect, leading to inadequate statistical power to detect the interaction. In this review, I will discuss the most common sources of bias in the estimation of gene $\mathrm{x}$ lifestyle interactions, and will discuss how such factors could be addressed in the future to enhance our potential to identify and replicate interactions for cardiometabolic diseases.
\end{abstract}

Keywords Gene · Polymorphism · Genetic variant . Environment $\cdot$ Lifestyle $\cdot$ Physical activity $\cdot$ Diet $\cdot$ Interaction . Bias · Misclassification · Confounding · Systematic error . Random error $\cdot$ Recall bias $\cdot$ FTO $\cdot$ Body mass index · Meta-analysis · Study design · Cross-sectional study · Prospective study $\cdot$ Epidemiology $\cdot$ Cardiometabolic disease Obesity · Type 2 diabetes · Cardiovascular disease

\section{Introduction}

Cardiometabolic diseases, such as cardiovascular disease, diabetes and obesity, are the leading causes of morbidity and mortality and represent one of the biggest challenges for

\section{T. O. Kilpeläinen $(\triangle)$}

The Novo Nordisk Foundation Center for Basic Metabolic Research, Section of Metabolic Genetics, Faculty of Health and Medical

Sciences, University of Copenhagen,

Universitetsparken 1, 2100 Copenhagen Ø, Denmark

e-mail: tuomas.kilpelainen@sund.ku.dk health care systems worldwide. Currently, more than 500 million adults are estimated to be obese [1], nearly 400 million have type 2 diabetes [2] and an estimated 17 million people die of cardiovascular disease every year [3]. Furthermore, a dramatic increase in the cardiometabolic disease burden has been predicted over the next two decades [2], underlining the need to develop better preventive strategies.

In the efforts to prevent the global epidemic of cardiometabolic diseases, it is essential to gain deeper knowledge on the biological mechanisms that lead to these common disorders. While lifestyle clearly affects a person's risk of developing cardiometabolic disease, there is also substantial evidence of a strong heritable component. Based on family and twin studies, approximately half of the risk of obesity, type 2 diabetes, and cardiovascular disease is due to genetic factors [4-7]. The genetic predisposition is, however, modified by lifestyle, a concept which is broadly referred to as gene $\mathrm{x}$ lifestyle interaction. By its epidemiological definition, a gene $\mathrm{x}$ lifestyle interaction is said to occur when there is a mutual dependency between a genetic variant and a lifestyle factor, such that the magnitude of the variant's effect on the outcome depends on the level of the lifestyle factor, and vice versa. Thereby, a genetic variant may strongly increase disease risk in a specific subgroup of individuals, for example in persons who are sedentary (Fig. 1), whereas its influence in other individuals is attenuated, abolished, or even reversed.

The past few years have seen tremendous progress in our understanding of the genetic basis of cardiometabolic diseases. Hundreds of novel susceptibility loci have been identified by the genome-wide association study approach [8-13]; a hypothesisfree method where hundreds of thousands of genetic variants across human genome are tested in large population samples to reveal the strongest genetic signals associated with the trait of interest. However, in contrast to the fast-paced progress in mapping the risk variants for cardiometabolic diseases, the success in identifying interactions between genetic variants 


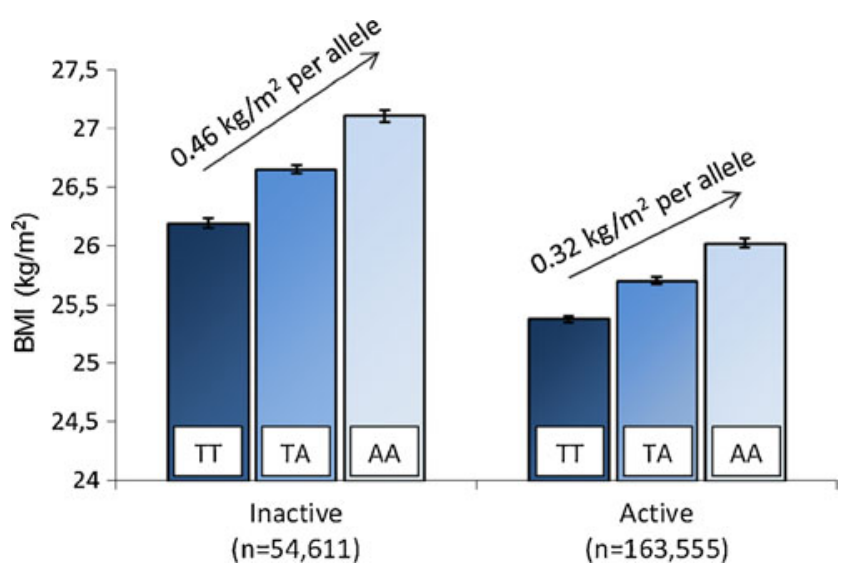

Fig. 1 The association of the FTO obesity risk variant with body mass index in the inactive and physically active groups in a meta-analysis of 218,166 adults. The genotypes indicate the homozygous common allele carriers (TT), heterozygous genotype carriers (TA) and homozygous minor allele carriers (AA) of the rs 9939609 polymorphism in the FTO gene. Adapted from [24]

and lifestyle has been much more limited. Although many examples of gene $\mathrm{x}$ lifestyle interactions have been published, typically in small-scale and likely underpowered samples, very few of the findings have been adequately replicated in independent studies, and the established interaction effect sizes have been too small to be clinically relevant $[14,15 \cdot]$. This suggests that gene $\mathrm{x}$ lifestyle interaction effects may, in general, be small in magnitude and/or more complex than initially thought.

Although the demand for a larger sample size to identify an interaction effect as compared to genetic main effects [16, 17], as well as the potential complexity of interaction effects [18••], may largely explain the slow pace of discoveries, the progress in the field may also be hindered by a tendency of epidemiological studies to underestimate the true interaction effect sizes [19]. As sample size requirements increase nonlinearly when interaction effects become closer to the null value, any factor that leads to a deflation in the observed effect size may make the detection of an interaction effect unfeasible, even when very large population samples are available for the analysis. Being able to estimate the effect sizes for gene $\mathrm{x}$ lifestyle interactions more accurately could increase our chances of identifying interactions, and potentially upgrade the relevance of the findings for public health. In this paper, I will review the most common sources of bias in the analysis of gene $\mathrm{x}$ lifestyle interactions, and will discuss how these factors could be accounted for in future studies to enhance our ability to identify gene $\mathrm{x}$ lifestyle interactions for cardiometabolic diseases.

\section{Common Sources of Bias in Studies of Gene $\times$ Lifestyle Interactions}

For the purposes of this review, I will include examples of bias in studies of gene $\mathrm{x}$ lifestyle interactions by making observations from a published, large-scale meta-analysis of the interaction between FTO obesity locus and physical activity (referred to as the FTOxPA study), which I will first briefly summarize here.

The locus in the first intron of the FTO gene represents the strongest known susceptibility locus for common obesity; each minor allele of the risk-variant increases body weight by $\sim 1 \mathrm{~kg}$ [20-22]. Soon after the initial discovery of FTO in 2007, a cross-sectional analysis of 5,554 Danish individuals showed an interaction between the FTO locus and physical activity on the level of body mass index (BMI), indicating that the BMIincreasing effect of the minor allele may be attenuated by physical activity [23]. A number of subsequent studies followed-up this finding, but with inconsistent results, raising doubts about the veracity of the interaction. To firmly confirm or refute the hypothesis that physical activity attenuates the BMI-increasing effect of the FTO locus, we invited all studies with data on FTO genotypes and physical activity to participate in a meta-analysis [24]. Importantly, although not discussed further in this review, the approach of pooling together both published and unpublished studies was crucial to avoid publication bias resulting from the possibility that non-significant interactions may often not be reported in the literature [25•*]. The final meta-analysis included 45 studies, in which physical activity was assessed in various ways, and it was therefore centrally standardized into a dichotomous trait (inactive vs. physically active) within each participating study. Consistent with the early findings, our meta-analysis of 218,166 adults confirmed that the BMI-increasing effect of the locus in the first intron of the FTO gene is smaller in people with physically active lifestyle than in those being sedentary; the effect was attenuated by approximately a third (by $\sim 0.4 \mathrm{~kg}$ per minor allele) in active as compared to inactive individuals (Fig. 1). There was, however, heterogeneity in the interaction effect $\left(I^{2}=36 \%\right)$, and further analyses indicated that the geographic origin of the participating studies was a major source of this heterogeneity. The interaction was almost entirely driven by North American cohorts, whereas in European cohorts, the effect size for interaction was close to zero. Apart from the meta-analysis of adults, we also performed a meta-analysis of nine pediatric cohorts, but found no evidence of interaction in these 19,268 children or adolescents.

\section{Error in the Measurement of Lifestyle}

The recent technological developments have enabled fast and accurate measurement of genotypes for large sample sizes in a standardized manner. However, the accurate and precise assessment of the lifestyle component of gene $\mathrm{x}$ lifestyle interactions remains a major challenge in epidemiological studies. In most cases, limitations in time and expenses, or the lack of appropriate methods and technologies, necessitate the use of assessment tools that are based on participant's self-reporting of lifestyle behaviors via questionnaires, rather than direct, 
objective methods that are generally more precise [26, 27]. In the FTOxPA study, which aimed to include all existing data, only three out of 45 participating studies had measured physical activity objectively (with an accelerometer or a heart rate sensor), whereas other studies had assessed physical activity by a self-report instrument [24]. The low precision and accuracy of most self-report-based assessments leads to a large error in the measurement of the lifestyle exposure, which can either be random or systematic error.

With random error, there is a difference between the true value of the lifestyle exposure and the recorded value, which is due to chance alone, so that the measurements fluctuate unpredictably around their true values [28••]. Therefore, we expect that the mean derived from a large number of observations is accurate, but that the precision of the estimate is low; the latter can be mitigated by increasing sample size to decrease the standard error of the mean estimate. In a regression analysis of exposure-outcome relationship, random measurement error in the outcome variable can indeed be compensated for either by increasing the sample size or the number of outcome measurements taken per individual. However, the same does not apply when the measurement error is in the exposure variable. A random error in the exposure measurement will bias the effect size for the association between the exposure and the outcome toward the zero (regression dilution bias). Increasing sample size will thus improve precision, but the effect estimate will remain inaccurate [28••]. As a simple example, in the FTOxPA study, we used a standardized physical activity variable across all studies, where the individuals were stratified into 'inactive' and 'physically active' subgroups [24]. In the ideal scenario, all individuals with physical activity levels corresponding to the definition of 'inactive' will be classified as 'inactive' (on average $25 \%$ of individuals), and all other individuals will be classified as 'active'. However, due to misclassification of physical activity, some of the individuals who are, in reality, 'inactive', will be classified as 'active', and vice versa. This random misclassification will mask some of the true difference in BMI between the physical activity strata. If the genotype and physical activity variables are independent and the misclassification is independent on the genotype, this misclassification is likely to attenuate the interaction effect size between FTO and physical activity [19, 29]. However, a bias increasing the interaction effect size is also possible depending on the true underlying interaction model [19, 29].

In systematic error the deviations are consistently wrong in a particular direction so that they overestimate or underestimate the true values, which can either produce spurious differences or mask the true differences. Systematic error in the assessment of lifestyle is often due to recall bias. For instance, in the FTOxPA study, obese individuals may have systematically over-reported their engagement in physical activities [30], which would overall lead to an inflated number of the carriers of the BMI-increasing allele of FTO being classified as 'physically active'. This would attenuate the true differences in the observed effect of the BMI-increasing allele of FTO between the 'inactive' and 'active' groups; i.e., lead to an underestimation of the interaction effect size. The impact of recall bias is likely to be most apparent when gene $\mathrm{x}$ lifestyle interactions are studied using a case-control design for the risk of a manifest disease, such as type 2 diabetes or cardiovascular disease, for which the clinical management of the condition typically involves education and advice about lifestyle. When the researchers look back in time to ascertain each person's exposure status, the individuals who have had the disease tend to remember exposures to putative lifestyle risk factors more strongly than controls, which exaggerates the relation between the lifestyle exposure and the disease risk and may bias the interaction estimate either toward or away from the null [31].

An increase in the measurement precision of a lifestyle exposure can have a dramatic impact on the statistical power to detect a gene $\mathrm{x}$ lifestyle interaction [17], which underscores the importance of precise measurement of lifestyle. Although statistical correction methods can be used to adjust for random measurement error if correction factors from internal validation studies are available, these methods do little in terms of recovering lost statistical power [32•] and rely on assumptions that may often not be met or are difficult to confirm [33]. The best strategy is to reduce the error at the study design stage by using measurement instruments that yield precise estimates and/or by collecting repeated measurements for each participant. Objective methods are advantageous as they remove many of the issues of recall bias, and the level of measurement precision is generally an order of magnitude greater than for self-report instruments [26, 27]. These methods may however be affected by the Hawthorne effect, where individuals alter their behavior in response to being observed [34]. Furthermore, objective methods are often time and cost-intensive and may excessively burden the respondent, which makes it difficult to apply them in large-scale epidemiological studies. Recent technological advancements, such as mobile information technologies, may provide novel opportunities to improve the assessment and reduce the costs associated with the collection and processing of lifestyle data [35, 36]. As more epidemiological studies apply objective methods for assessing lifestyle behaviors, meta-analyses of these studies may soon provide a well-powered platform for studies of gene $\mathrm{x}$ lifestyle interactions.

When self-report-based instruments are used for assessing lifestyle in studies with discrete disease outcomes, the minimization of recall bias requires prospective data, where lifestyle has been assessed prior to the onset of disease. It is, however, often difficult to amass large sample collections of incident cases, that also include precisely assessed exposure and outcome data, to be adequately powered to detect interactions. An efficient approach to reach these aims is a nested 
case-control study within an existing large cohort. This approach has been adopted, for example, in the InterAct project, a nested case-cohort study of more than 28,000 incident diabetics and non-diabetics selected from the European Investigation into Cancer and Nutrition (EPIC) study [37]. Alternatively, post-hoc analyses in existing randomized controlled trials of lifestyle modification can be performed [38-40], but again, it is difficult to reach a sufficiently large sample size for such studies to be adequately powered. As clinical trials often differ substantially in design and study populations, pooling data from multiple trials might reduce rather than increase statistical power. A third possibility, although costly, is to design new studies focusing specifically on gene $\mathrm{x}$ lifestyle interactions, in which recruitment by genotype is used to enrich genetic effects at the extremes of the genotype distribution [14]. No examples of such studies have yet been reported in the literature.

\section{Modeling and Harmonization of Lifestyle Exposures}

As the effect sizes for gene $\mathrm{x}$ lifestyle interactions on cardiometabolic traits are likely to be small, their detection will require very large sample sizes [16, 17]. Accumulating a sufficient sample size will often require pooling multiple large-scale studies into a meta-analysis. Unless consistent methods of characterizing lifestyle have been used across all participating studies (e.g. all studies participating in the InterAct project used standardized EPIC self-report questionnaires [37]), the differences in assessment tools and population characteristics necessitate meticulous harmonization of lifestyle exposures to minimize heterogeneity across the participating studies. For complex multidimensional behaviors, such as physical activity or diet, this is far from a straightforward task. The modeling of physical activity as the exposure variable, for example, precludes considerations of how various dimensions of physical activity are incorporated in the variable, including the context (leisure-time, occupation, transportation), mode (e.g., walking, tennis), frequency, duration, and intensity of physical activity. Furthermore, we may choose to model either a categorical or a continuously distributed physical activity variable that may be based, for instance, on energy expenditure, duration of activity, or an activity score. As the stratification of a normally distributed exposure variable nearly always reduces the power to detect an interaction, the use of a continuous physical activity trait is generally recommendable [41]. However, heterogeneity in the measurement tools may often necessitate the standardization of physical activity into a categorical variable, which precludes further considerations of the number of strata as well as the boundaries separating the strata, that can either be distributionbased (e.g., tertiles or quantiles) or based on absolute values of physical activity. Each of these specifications may have a major impact on the observed interaction effect size and its error estimate.

In the FTOxPA study, we standardized physical activity into a simple dichotomous variable, 'inactive' vs. 'active', in all studies [24]. The group 'inactive' included all sedentary individuals (defined as a sedentary occupation and $<1$ hours per week of commuting or leisure-time moderate-to-vigorous physical activity) and the group 'active' involved all remaining individuals. Although the standardization of the physical activity variable could have been carried out in other ways (e.g., by using the median of physical activity as the cutoff, or by forming three physical activity strata), three arguments supported the selection of the 'inactive' vs. 'active' dichotomization for physical activity: (1) previous studies that had shown a significant interaction between FTO and physical activity had compared sedentary individuals to other individuals (e.g., [23]); (2) the benefits of increased physical activity in improving health and fitness outcomes are generally most apparent at the lower tail of the distribution of physical activity, i.e., when a sedentary person increases his/ her habitual activity levels, whereas smaller benefits are typically achieved at higher baseline levels of physical activity [42]; (3) In nearly all studies that had used questionnairebased assessment of physical activity, the questions clearly identified sedentary individuals [24], whereas in higher activity levels, an attempt to harmonize the categories would have likely resulted in highly heterogeneous strata. We thus expected that the stratification into sedentary and other individuals would be feasible and retain a fair degree of homogeneity of the physical activity categories across all studies, while still providing a sufficient difference between the physical activity strata to expose an underlying interaction.

The studies performed of gene $\mathrm{x}$ lifestyle interactions so far have not provided information on the dose-response relationship between the level of the lifestyle exposure and the genetic effect, which may have important implications for optimizing the power of an interaction study. More specifically, if a simple dichotomous exposure variable is used, such as in the FTOxPA study, the interaction effect size depends on how 'ideal' the cut-off separating the physical activity strata is with regard to the dose-response relationship between the level of lifestyle exposure and the size of the additive genetic effect. In the efforts to maximize the statistical power for detecting an interaction, the ideal cut-off point would be where the largest difference in the genetic main effects (i.e., the strongest interaction effect size) is seen between the physical activity strata, and where both physical activity strata still have sufficiently large sample sizes to allow robust regression analysis and to produce modest standard error for the interaction effect estimate. That being said, when categorizing exposure variables, it is critical to form the hypotheses that guide the choice of boundaries in advance to avoid false positive findings produced by data dredging [43]. 
In the FTOxPA study, heterogeneity between studies in the absolute cut-off points separating the physical activity strata may have contributed to the larger interaction effect size observed in North American as compared to European cohorts. This heterogeneity is partly due to population-specific characteristics, such as the distribution of physical activity levels, but also due to differences in the assessment tools for physical activity. In particular, there may be lower average levels of physical activity and higher prevalence of very sedentary individuals in North American than in European populations [44, 45], and all North American studies had quantified physical activity using a continuously distributed physical activity variable, whereas most European studies used categorical variables [24]. Indeed, in meta-analyses of gene $\mathrm{x}$ lifestyle interactions, the approach of pooling multiple cohorts with diverse assessments of the exposure may sometimes be counter-productive, owing to the heterogeneity between the populations and assessment tools, and the low power incurred when meta-analyzing data from studies that lack standardized measures. Therefore, before embarking in a meta-analysis effort, it is worth considering whether a focus on fewer, more homogenous population studies with wellmeasured lifestyle variables, rather than many heterogeneous studies of variable quality, will be the optimal approach.

\section{Unmeasured Confounders or Effect Modifiers}

The conventional modeling of interactions for one specific lifestyle factor at a time in a linear or logistic regression model may be overly simplistic [18••], and the observed interaction may depend on a constellation of lifestyle and other risk factors. Such unknown or unmeasured confounders (variables associated with both the exposure and the outcome) or effectmodifiers (variables that interact with the exposure $\mathrm{x}$ SNP interaction term, leading to a three-way or higher order interaction) may lead to a bias in the observed interaction effect, which, in a meta-analysis of gene $\mathrm{x}$ lifestyle interactions, will introduce between-study heterogeneity in the observed effect estimates. For example, physical activity is likely to be a marker of a generally healthy lifestyle, and other effect modifiers, such as specific dietary factors [46-48], may have contributed the observed interaction effect between FTO and physical activity in the FTOxPA study. Furthermore, cultural differences in lifestyle may lead these factors to be more strongly correlated with physical activity in North American than in European studies, which could partly explain the difference in the observed interaction effect size between these two geographic regions.

A recently implemented method for identifying genetic variants that are sensitive to the environment overall and thus to prioritize variants for interaction studies with specific lifestyle or other environmental factors, is a genetic association study of phenotypic variance [49]. As any environment is likely to be heterogenous, so that the environment experienced by each individual differs, a gene $\mathrm{x}$ environment interaction may manifest as differences in the variance of a phenotype between the genotype groups. Thus, even if the environment is not directly measured, the method gives an indication of interaction between a genetic variant and the environment [50]. A genome-wide association study of the phenotypic variance of BMI in $\sim 170,000$ individuals identified FTO as the strongest finding, giving further evidence of this locus with the environment [51]. To resolve which specific lifestyle or environmental components alone or in combination 'causally' interact with FTO, a large-scale intervention study or mechanistic studies will be required.

\section{Temporality of Exposures}

Interactions are often studied using a cross-sectional study design, but as these studies only offer a snapshot of a single moment in time, they do not give information on the longitudinal relationships between variables, and cannot establish whether a causal relationship underlies an interaction effect. Thus, even if the FTOxPA study showed an interaction between FTO and physical activity in cross-sectional data, we were not able to tease out whether physical activity inflicts a change in the genetic effect, and it remains possible that there is only a correlation but no causal relationship between physical activity and the BMI-increasing effect of FTO. Longitudinal studies with repeated observations over time are required to observe the temporal order of events and to ascertain causal effects. Furthermore, interactions between genetic loci and lifestyle behaviors may appear at different stages of life and under differing circumstances [52], and only studies with long follow-up may provide information on the timing of interaction effects during life course. In the FTOxPA study, our metaanalysis of 19,268 children and adolescents showed a null effect. Although the absence of interaction may be explained by the multiple sources of bias as discussed in this review along with a lack of statistical power, the results may also indicate that the interaction effect between FTO and physical activity becomes apparent only in adulthood [24]. Ultimately, to estimate the true magnitude of change in genetic effect induced by a specific change in the lifestyle exposure in a target population, a large-scale randomized controlled trial will be required.

\section{Conclusion}

Despite the major progress in our understanding of the genetic basis of cardiometabolic disease during recent years, there has been relatively little advancement in our knowledge of gene $\mathrm{x}$ lifestyle interactions in the development of these common disorders. Apart from being challenged by the generally small 
effect sizes of interactions, the progress in the field may be hindered by biases stemming from the simplistic modeling of potentially complex interaction effects, and the difficulties in assessing multidimensional lifestyle behaviors, such as physical activity and diet. Technological advancements in the measurement of complex lifestyle behaviors, along with the deployment of omics-techniques and insightful modeling of interactions within large-scale biobank efforts with longitudinal data, might soon lead to a flux of novel discoveries. Such developments may also help to determine the true, underlying interaction effect sizes that are crucial when considering the potential translational implications of gene $\mathrm{x}$ lifestyle interactions.

\section{Compliance with Ethics Guidelines}

Conflict of Interest Tuomas O. Kilpeläinen declares that he has no conflict of interest.

Human and Animal Rights and Informed Consent This article does not contain any studies with human or animal subjects performed by any of the authors.

\section{References}

Papers of particular interest, published recently, have been highlighted as:

- Of importance

-. Of major importance

1. Finucane MM, Stevens GA, Cowan MJ, et al. National, regional, and global trends in body-mass index since 1980: systematic analysis of health examination surveys and epidemiological studies with 960 country-years and 9.1 million participants. Lancet. 2011;377:557-67.

2. Whiting DR, Guariguata L, Weil C, Shaw J. IDF diabetes atlas: global estimates of the prevalence of diabetes for 2011 and 2030. Diabetes Res Clin Pract. 2011;94:311-21.

3. The Global Burden of Disease: 2004 update. Geneva, Switzerland: World Health Organization; 2008.

4. Maes HH, Neale MC, Eaves LJ. Genetic and environmental factors in relative body weight and human adiposity. Behav Genet. 1997;27:325-51.

5. Poulsen P, Kyvik KO, Vaag A, Beck-Nielsen H. Heritability of type II (non-insulin-dependent) diabetes mellitus and abnormal glucose tolerance-a population-based twin study. Diabetologia. 1999;42:139-45.

6. Zdravkovic S, Wienke A, Pedersen NL, de Faire U. Genetic influences on angina pectoris and its impact on coronary heart disease. Eur J Hum Genet. 2007;15:872-7.

7. Zdravkovic S, Wienke A, Pedersen NL, et al. Heritability of death from coronary heart disease: a 36-year follow-up of 20966 Swedish twins. J Intern Med. 2002;252:247-54.

8. Morris AP, Voight BF, Teslovich TM, et al. Large-scale association analysis provides insights into the genetic architecture and pathophysiology of type 2 diabetes. Nat Genet. 2012;44:981-90.

9. Scott RA, Lagou V, Welch RP, et al. Large-scale association analyses identify new loci influencing glycemic traits and provide insight into the underlying biological pathways. Nat Genet. 2012;44:991-1005.
10. Speliotes EK, Willer CJ, Berndt SI, et al. Association analyses of 249, 796 individuals reveal 18 new loci associated with body mass index. Nat Genet. 2010;42:937-48.

11. Teslovich TM, Musunuru K, Smith AV, et al. Biological, clinical and population relevance of 95 loci for blood lipids. Nature. 2010;466:707-13.

12. Traylor M, Farrall M, Holliday EG, et al. Genetic risk factors for ischaemic stroke and its subtypes (the METASTROKE collaboration): a meta-analysis of genome-wide association studies. Lancet Neurol. 2012;11:951-62.

13. Wain LV, Verwoert GC, O'Reilly PF, et al. Genome-wide association study identifies six new loci influencing pulse pressure and mean arterial pressure. Nat Genet. 2011;43:1005-11.

14. Franks PW. Gene $\mathrm{x}$ environment interactions in type 2 diabetes. Curr Diab Rep. 2011;11:552-61.

15. - Joseph PG, Pare G, Anand SS. Exploring gene-environment relationships in cardiovascular disease. Can J Cardiol. 2013;29:37-45. The most recent literature review of studies of gene $x$ environment interactions in cardiometabolic disease.

16. Smith PG, Day NE. The design of case-control studies: the influence of confounding and interaction effects. Int $\mathrm{J}$ Epidemiol. 1984;13:356-65.

17. Wong MY, Day NE, Luan JA, et al. The detection of geneenvironment interaction for continuous traits: should we deal with measurement error by bigger studies or better measurement? Int $\mathrm{J}$ Epidemiol. 2003;32:51-7.

18. •- Aschard H, Lutz S, Maus B, et al. Challenges and opportunities in genome-wide environmental interaction (GWEI) studies. Hum Genet. 2012;131:1591-613. Discusses the strategies and methodological aspects of identifying gene $x$ environment interactions .

19. Garcia-Closas M, Rothman N, Lubin J. Misclassification in casecontrol studies of gene-environment interactions: assessment of bias and sample size. Cancer Epidemiol Biomarkers Prev. 1999;8:1043-50.

20. Dina C, Meyre D, Gallina S, et al. Variation in FTO contributes to childhood obesity and severe adult obesity. Nat Genet. 2007;39:724-6.

21. Frayling TM, Timpson NJ, Weedon MN, et al. A common variant in the FTO gene is associated with body mass index and predisposes to childhood and adult obesity. Science. 2007;316:889-94.

22. Scuteri A, Sanna S, Chen WM, et al. Genome-wide association scan shows genetic variants in the FTO gene are associated with obesityrelated traits. PLoS Genet. 2007;3:e115.

23. Andreasen CH, Stender-Petersen KL, Mogensen MS, et al. Low physical activity accentuates the effect of the FTO rs 9939609 polymorphism on body fat accumulation. Diabetes. 2008;57:95-101.

24. Kilpelainen TO, Qi L, Brage S, et al. Physical activity attenuates the influence of FTO variants on obesity risk: a meta-analysis of 218,166 adults and 19,268 children. PLoS Med. 2011;8:e1001116.

25. • Palla L, Higgins JP, Wareham NJ, Sharp SJ. Challenges in the use of literature-based meta-analysis to examine gene-environment interactions. Am J Epidemiol. 2010;171:1225-32. Discusses the challenges of performing a literature-based meta-analysis of gene $x$ environment interactions and highlights the biases that such metaanalyses are prone to.

26. Helmerhorst HJ, Brage S, Warren J, et al. A systematic review of reliability and objective criterion-related validity of physical activity questionnaires. Int J Behav Nutr Phys Act. 2012;9:103.

27. Jenab M, Slimani N, Bictash M, et al. Biomarkers in nutritional epidemiology: applications, needs and new horizons. Hum Genet. 2009;125:507-25.

28. • Hutcheon JA, Chiolero A, Hanley JA. Random measurement error and regression dilution bias. BMJ. 2010;340:c2289. Describes the impact of random error in a regression analysis of exposure-outcome association and discusses approaches for dealing with it.

29. Lundberg M, Hallqvist J, Diderichsen F. Exposure-dependent misclassification of exposure in interaction analyses. Epidemiology. 1999;10:545-9. 
30. Pietilainen KH, Korkeila M, Bogl LH, et al. Inaccuracies in food and physical activity diaries of obese subjects: complementary evidence from doubly labeled water and co-twin assessments. Int J Obes (Lond). 2010;34:437-45.

31. Schulz KF, Grimes DA. Case-control studies: research in reverse. Lancet. 2002;359:431-4.

32. - Freedman LS, Schatzkin A, Midthune D, Kipnis V. Dealing with dietary measurement error in nutritional cohort studies. J Natl Cancer Inst. 2011;103:1086-92. Describes the impact of dietary measurement error in studies of diet-disease associations and discusses approaches for dealing with it.

33. Frost C, White IR. The effect of measurement error in risk factors that change over time in cohort studies: do simple methods overcorrect for 'regression dilution'? Int J Epidemiol. 2005;34:1359-68.

34. McCarney R, Warner J, Iliffe S, et al. The Hawthorne Effect: a randomised, controlled trial. BMC Med Res Methodol. 2007;7:30.

35. Gurrin C, Qiu Z, Hughes M, et al. The smartphone as a platform for wearable cameras in health research. Am J Prev Med. 2013;44:308-13.

36. Thompson FE, Subar AF, Loria CM, et al. Need for technological innovation in dietary assessment. J Am Diet Assoc. 2010;110:48-51.

37. Langenberg C, Sharp S, Forouhi NG, et al. Design and cohort description of the InterAct Project: an examination of the interaction of genetic and lifestyle factors on the incidence of type 2 diabetes in the EPIC Study. Diabetologia. 2011;54:2272-82.

38. Jablonski KA, McAteer JB, de Bakker PI, et al. Common variants in 40 genes assessed for diabetes incidence and response to metformin and lifestyle intervention in the diabetes prevention program. Diabetes. 2010;59:2672-81.

39. Kilpelainen TO, Lakka TA, Laaksonen DE, et al. SNPs in PPARG associate with type 2 diabetes and interact with physical activity. Med Sci Sports Exerc. 2008;40:25-33.

40. Qi Q, Bray GA, Smith SR, et al. Insulin receptor substrate 1 gene variation modifies insulin resistance response to weightloss diets in a 2-year randomized trial: the Preventing Overweight Using Novel Dietary Strategies (POUNDS LOST) trial. Circulation. 2011;124:563-71.
41. Naggara O, Raymond J, Guilbert F, et al. Analysis by categorizing or dichotomizing continuous variables is inadvisable: an example from the natural history of unruptured aneurysms. AJNR Am J Neuroradiol. 2011;32:437-40.

42. Haskell WL, Lee IM, Pate RR, et al. Physical activity and public health: updated recommendation for adults from the American College of Sports Medicine and the American Heart Association. Med Sci Sports Exerc. 2007;39:1423-34.

43. Turner EL, Dobson JE, Pocock SJ. Categorisation of continuous risk factors in epidemiological publications: a survey of current practice. Epidemiol Perspect Innov. 2010;7:9.

44. Friedenreich CM, Courneya KS, Neilson HK, et al. Reliability and validity of the Past Year Total Physical Activity Questionnaire. Am J Epidemiol. 2006;163:959-70.

45. Hagstromer M, Troiano RP, Sjostrom M, Berrigan D. Levels and patterns of objectively assessed physical activity-a comparison between Sweden and the United States. Am J Epidemiol. 2010;171:1055-64.

46. Ahmad T, Lee IM, Pare G, et al. Lifestyle interaction with fat mass and obesity-associated (FTO) genotype and risk of obesity in apparently healthy U.S. women. Diabetes Care. 2011;34:675-80.

47. Sonestedt E, Gullberg B, Ericson U, et al. Association between fat intake, physical activity and mortality depending on genetic variation in FTO. Int J Obes (Lond). 2011;35:1041-9.

48. Sonestedt E, Roos C, Gullberg B, et al. Fat and carbohydrate intake modify the association between genetic variation in the FTO genotype and obesity. Am J Clin Nutr. 2009;90:1418-25.

49. Pare G, Cook NR, Ridker PM, Chasman DI. On the use of variance per genotype as a tool to identify quantitative trait interaction effects: a report from the Women's Genome Health Study. PLoS Genet. 2010;6:e1000981.

50. Hill WG, Mulder HA. Genetic analysis of environmental variation. Genet Res (Camb). 2010;92:381-95.

51. Yang J, Loos RJ, Powell JE, et al. FTO genotype is associated with phenotypic variability of body mass index. Nature. 2012;490:267-72.

52. Mathers JC. Nutritional modulation of ageing: genomic and epigenetic approaches. Mech Ageing Dev. 2006;127:584-9. 